\title{
Generation of THz Radiation by Sampled Grating DBR Mode Locked Laser Diodes
}

\author{
Lianping Hou ${ }^{1 *}$, Bin Hou ${ }^{1}$, Song Liang ${ }^{2}$, Dejun Chen ${ }^{3}$, John H. Marsh ${ }^{1}$ \\ ${ }^{I}$ School of Engineering, University of Glasgow, Glasgow, G12 8QQ, U.K \\ ${ }^{2}$ Institute of Semiconductors, Chinese Academy of Sciences, No. A35, East Qinghua Road, Haidian District, Beijing 100083, China \\ ${ }^{3}$ School of Optoelectronic Information, University of Electronic Science and Technology of China, Chengdu 610054, China \\ *Corresponding author: lianping.hou@glasgow.ac.uk
}

\begin{abstract}
GHz} \mathrm{THz}$ signals were generated based on $1.55 \mu \mathrm{m}$ operating wavelength sampled grating distributed-Bragg-reflector mode locked laser diodes and photomixing techniques which will pave a way to provide compact low-cost, robust THz sources.

OCIS codes: (140.4050) Mode-locked lasers; (040.2235) Far infrared or terahertz; (320-7090) Ultrafast lasers.
\end{abstract}

Systems based on $\mathrm{THz}$ radiation are continuing to be developed for a wide range of applications, with $\mathrm{THz}$ photonics becoming established as a key technology [1]. Until recently there has been comparatively little exploitation of $\mathrm{THz}$ radiation due to the lack of sources and detectors, and, for many systems, a compact low-cost source of THz radiation is vitally important. Ultrafast $1.55 \mu \mathrm{m}$ semiconductor mode-locked laser diodes (SMLLDs) operating at Terahertz repetition frequencies $\left(F_{r}\right)$ - open exciting opportunities in THz signal generation because of their miniature form factor, inherent robustness and the fact that erbium-doped fiber amplifiers (EDFAs) can be used for subsequent amplification. Such a system offers many benefits over the traditional method of $\mathrm{THz}$ generation using optical rectification and the linear electro-optic effect, which typically requires a Ti:Sapphire laser with the associated large system footprint and high power consumption.

Previously we have used conventional sampled grating distributed-Bragg-gratings (SGDBRs) as the reflectors to produce SMLLDs operating at $F_{r}=640 \mathrm{GHz}$ [2]. These devices have been shown to offer far superior reproducibility, controllability and wide operation range compared to all other reported types of $\mathrm{THz}$ frequency SMLLDs [3], such as colliding pulse mode locking (CPM) [4] and compound-cavity mode locking (CCM) devices $[5,6]$. In this paper, we use the $640 \mathrm{GHz}$ SGDBR SMLLDs as the source to pump a THz photoconductive antenna (PCA) to produce the THz signal. From the measured results, we can confirm that THz generation based on SGDBR SMLLDs offers the advantages in size, power requirements and robustness anticipated above, and which could pave the way for future developments in $\mathrm{THz}$ technology and practical applications.

The device structure and dimensions are the same as reported in [2], and are shown in Fig. 1(a). The saturable absorber (SA) section is $30 \mu \mathrm{m}$ long, the gain section is $960 \mu \mathrm{m}$ long, and the SGDBR section is $675 \mu \mathrm{m}$ long. Fig. 1(b) shows a schematic diagram of the $\mathrm{THz}$ signal generation setup based on the SGDBR SMLLDs and photomixing techniques. The $640 \mathrm{GHz}$ pulse train output from the device was amplified to an average power of 24 $\mathrm{mW}$ by the EDFA and then used to pump the THz PCA. The PCA was operated with a bias from the pulse generator switching between $0 \mathrm{~V}$ and $5 \mathrm{~V}$ at a frequency of $9 \mathrm{~Hz}$ and on a $50 \%$ duty cycle. A $640 \mathrm{GHz}$ band pass filter was placed between the PCA and the THz detector. The power of the THz signal was measured using a Golay cell and the lock-in amplifier with the $9 \mathrm{~Hz}$ reference modulation frequency supplied by the pulse generator. The currents applied to the gain and SGDBR sections, the voltage operated to the SA section and the measured THz signal power at each operation point were set or collected automatically by the personal computer (PC) MATLAB program.

Figure 2(a) shows the 2-dimensional optical spectra for a range of gain currents $\left(I_{\text {Gain }}\right)$ from $0 \mathrm{~mA}$ to $300 \mathrm{~mA}$, with the SA reverse voltage, $V_{S A}=-3.0 \mathrm{~V}$, and SGDBR current, $I_{S G D B R}=0 \mathrm{~mA}$. The laser shows stable operation

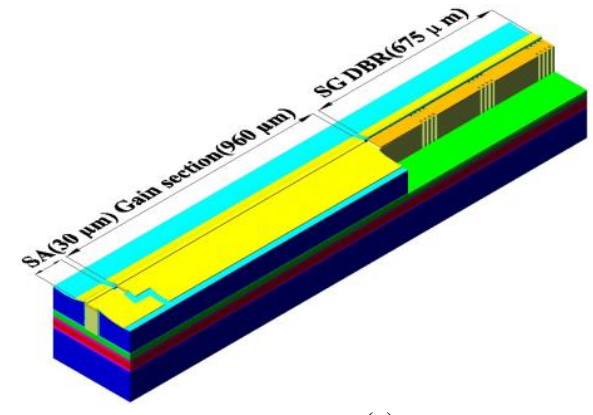

(a)

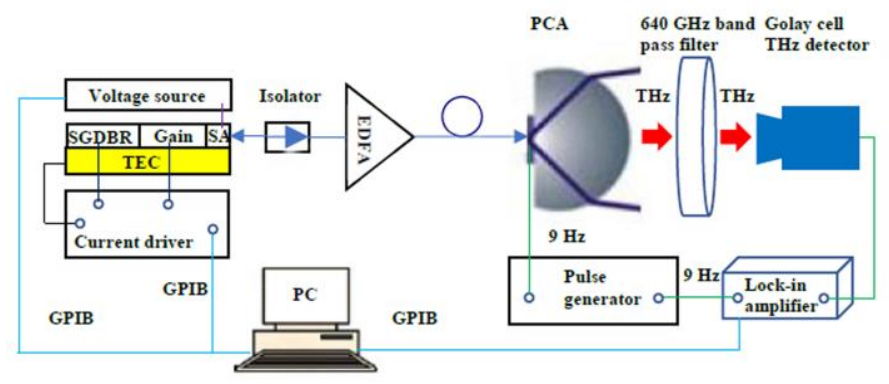

(b)

Fig.1 (a) Schematic diagram of the $1.55 \mu \mathrm{m}$ side-wall SGDBR SMLLD, and (b) schematic diagram of $640 \mathrm{THz}$ signal generation based on the $F_{r}=640 \mathrm{GHz}$ SGDBR SMLLD and PCA. 
with a mode spacing around $5.22 \mathrm{~nm}$. The optical spectrum at $I_{\text {Gain }}=204 \mathrm{~mA}$ is shown in Fig. 2(b). The central wavelength is $1567.70 \mathrm{~nm}$ with a channel spacing of $5.22 \mathrm{~nm}$ and a $3 \mathrm{~dB}$ bandwidth of $5.45 \mathrm{~nm}$. The measured autocorrelation (AC) trace is shown in Fig. 2(c), with the average period of the emitted pulse train measured as 1.57 ps, which corresponds to an $F_{r}$ of $637 \mathrm{GHz}$, consistent with the optical spectrum shown in Fig. 2(b). The AC width of an isolated pulse was $0.85 \mathrm{ps}$, which deconvolves to a pulse width of $0.55 \mathrm{ps}$, assuming a sech ${ }^{2}$ pulse shape. The time bandwidth product (TBP) of the pulse is equal to 0.37 , which is close to the transform-limited value $(\approx 0.315)$ of a sech ${ }^{2}$ pulse. Fig. 3 shows the measured 2-dimensional THz power map as a function of the $I_{\text {Gain }}$ and $V_{S A}$ in the cases of $I_{S G D B R}=0 \mathrm{~mA}$ and $10 \mathrm{~mA}$. The platform in the THz power, with a value around 40 arbitrary units, arises from amplified spontaneous emission noise from the EDFA itself. With the laser biased under mode locking conditions, significant $\mathrm{THz}$ powers above the noise floor were achieved across a wide operating range, i.e., $50 \mathrm{~mA} \leq$ $I_{\text {Gain }} \leq 300 \mathrm{~mA}$ and $-3 \mathrm{~V} \leq V_{S A} \leq 0 \mathrm{~V}$, but discontinuously. The largest continuous area can be seen for $I_{S G D B R}=0$ $\mathrm{mA}$, when $V_{S A}$ is between $-3 \mathrm{~V}$ and $-2.6 \mathrm{~V}$ and $I_{\text {Gain }}$ between $50 \mathrm{~mA}$ and $200 \mathrm{~mA}$. Increasing $I_{S G D B R}$ to $10 \mathrm{~mA}$ leads to a small reduction in the threshold current from $\sim 48 \mathrm{~mA}$ to $\sim 42 \mathrm{~mA}$, but the continuous areas of $\mathrm{THz}$ generation are smaller. Absolute calibration of the $\mathrm{THz}$ power is under way.

In conclusion, a THz signal of $640 \mathrm{GHz}$ was generated based on a $1.55 \mu \mathrm{m}$ SGDBR SMLLD, EDFA amplification and photomixing in a photoconductive antenna. The system is a compact low-cost and robust source of narrow-band $\mathrm{THz}$ radiation, with all components operating at room temperature. This approach is expected to open new applications of THz technology.

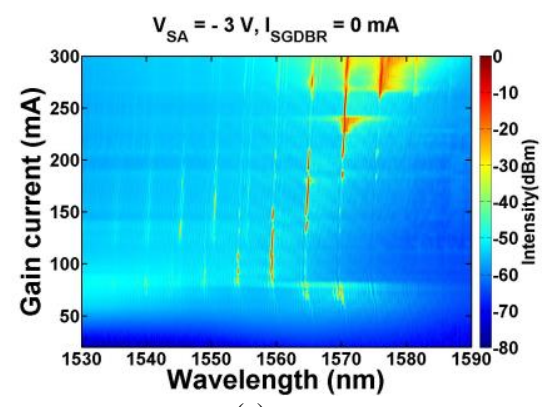

(a)

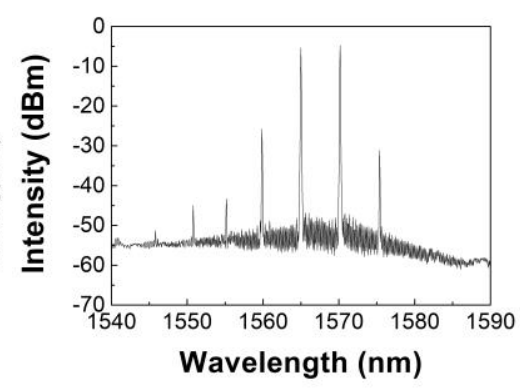

(b)

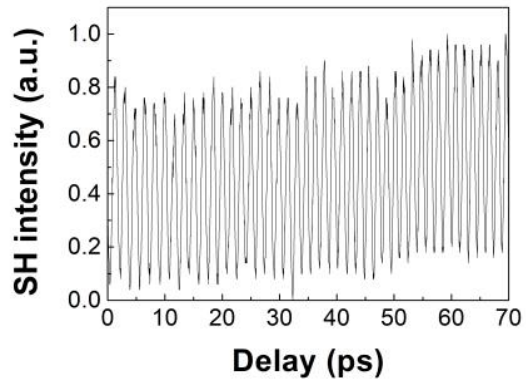

(c)

Fig. 2. (a) 2-Dimensional optical spectra measured as a function of the gain current at $I_{S G D B R}=0 \mathrm{~mA}$, (b) optical spectrum and (c) autocorrelation traces of the $640 \mathrm{GHz}$ SGDBR SMLLDs under $V_{S A}=-3 \mathrm{~V}, I_{\text {Gain }}=204 \mathrm{~mA}, I_{S G D B R}=0 \mathrm{~mA}$.

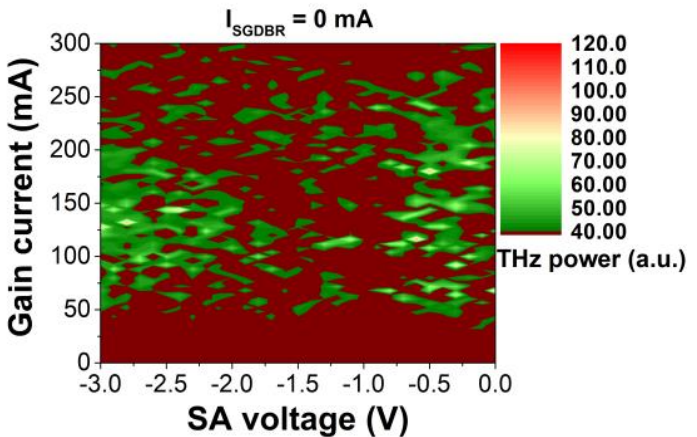

(a)

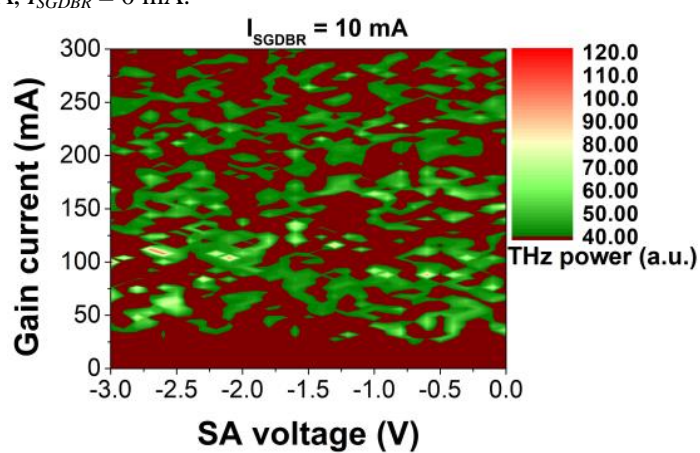

(b)

Fig. 3. Measured THz signal power as a function of the gain current and the SA reverse voltage at (a) $I_{S G D B R}=0 \mathrm{~mA}$, and (b) $10 \mathrm{~mA}$.

\section{References}

[1] M. Tonouchi, 'Cutting-edge terahertz technology,' Nature Photonics 1, 97 (2007).

[2] L. Hou, M. Haji, J. H. Marsh, 'Mode locking at Terahertz frequencies using a distributed Bragg reflector laser with a sampled grating,' Opt. Lett. 38, 1113-1115 (2013).

[3] J. H. Marsh and L. Hou, 'Mode-locked laser diodes and their monolithic integration,' IEEE J. Sel. Topics in Quantum Electron., 23, no. 6, Art. no. 1100611 (2017).

[4] T. Shimizu, I. Ogura, H. Yokoyama, '860 GHz rate asymmetric colliding pulse mode locked diode lasers,' Electron. Lett, 33, 1868-1869 (1997).

[5] S. Arahira, Y. Matsui, and Y. Ogawa, 'Mode-locking at very high repetition rates more than terahertz in passively mode-locked distributedBragg-reflector laser diodes,' IEEE J. Quantum Electron, 32, 1211-1224 (1996).

[6] D. A. Yanson, M. W. Street, S. D. McDougall, I. G. Thayne, J. H. Marsh, and E. A. Avrutin, 'Ultrafast harmonic mode-locking of monolithic compound-cavity laser diodes incorporating photonic-bandgap reflectors,' IEEE J. Quantum Electron. 38, 1-11 (2002). 